\title{
The Electron Microscope
}

\section{By Prof. L. C. Martin, Imperial College, London}

\section{INTRODUCTORY}

$I^{\mathrm{T}}$ has been known for many years that cathode rays can be deflected by electric and magnetic fields, but in 1926 Busch showed that axially symmetrical fields would have the power of focusing such rays, much as light is focused by a lens.

Any axially symmetrical lens system will produce a fairly sharp optical image of objects near the axis, provided that the aperture is so far reduced that only rays travelling very close to the axis are allowed to pass through the system, and provided moreover that strictly monochromatic light is employed. This reduction of the aperture is essential in general to avoid the spherical aberration of the rays which make larger angles with the axis, and the consequent deterioration of the definition of the image ; but on the other hand, the finite wave-length of light causes a spreading of each image point, such that the spread is inversely proportional to the aperture of the lens system. This tendency makes it impossible to retain very sharp images with optical lenses of smaller and smaller angular aperture, and highly complicated lens systems have to be used to allow of the attainment of large apertures without concurrent spherical aberration. Thus in the relatively simple case of the ultra-violet microscope objective (numerical aperture $1 \cdot 2$ ), five or six specially shaped lenses, mounted with the greatest care, have to be used; but the resolving limit cannot be brought much below about $0 \cdot 15 \mu$ without great difficulty. In other words, the closest approach of two object points compatible with the separation of their images is about one seven-thousandth part of a millimetre.

\section{Possibilities of the Electron Microscope}

Until the origin of the electron microscope, it did not seem possible that this limit would ever be lowered very considerably, but once the possibility of forming images with electrons was realized, it became apparent that further progress was possible. The experiments of Prof. G. P. Thomson and others had shown that the wavelengths of the 'waves' associated with mediumvelocity electrons are only about a hundredthousandth part of the wave-length of ultra-violet light, and therefore the relative aperture of an electron-optical system could be lowered drastically, in order to avoid spherical aberration, without incurring any considerable spreading of the elementary image points. Such 'lenses' have now been made which work at numerical apertures of much less than 0.1 ; but the residual spreading of the image is almost entirely due to other causes, that is, to 'chromatic aberration' (due to the

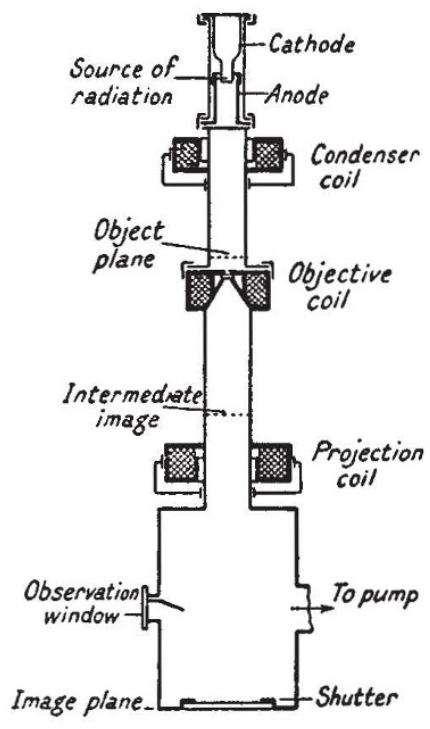

Fig. 1.

KNOLL AND RUSKA's ELECTRON MICROSCOPE.

presence of electrons with differing velocities), to spherical aberration, and to faulty centration together with the characteristic aberrations of extra. axial image points. The best of such images appear to have resolving limits of approximately $0.01 \mu$ and will bear magnifications up to 20,000 or more without losing apparent sharpness.

The pioneer work in building such instruments was due to Knoll and Ruska working in Berlin (1932). They employed a cold-cathode discharge tube as a source of electrons, which were projected through a very small aperture along the axis of a highly evacuated tube (Fig. 1). The electron beam passed in succession through ironclad coils representing the condenser, the objective, and the projection eyepiece of a microscope, and finally fell upon a fluorescent screen or photographic plate for the registration of the image. The first pictures obtained were 'silhouette' images of fibres, perforated metal foils, and the like. Then in Brussels, 
Marton made early experiments on the electronphotography of biological tissues impregnated with osmium, and of bacteria mounted on very thin films of nitro-cellulose. In Great Britain an instrument was designed by the present writer and his colleagues in co-operation with Messrs. Metropolitan-Vickers Electrical Co., Ltd., by whom it was made (Fig. 2). It is intended to give special facilities in the microscopy of objects alternatively by light or the electron beam, and experiments with it have been in progress for the past two years. The resolving limit attained is already better than that of the ultra-violet microscope, and Prof. G. P. Thomson is directing a systematic investigation of various troubles preventing better definition. Hot-cathode discharges are now being employed by the German workers.

The results so far obtained on the Continent are wonderfully promising, although claims have been made in a few cases which do not appear to be justified by the photographs reproduced. Exceptionally striking pictures of colloidal structures, for example, threads of nickel carbonyl, and particles of colloidal gold in a film of lithium

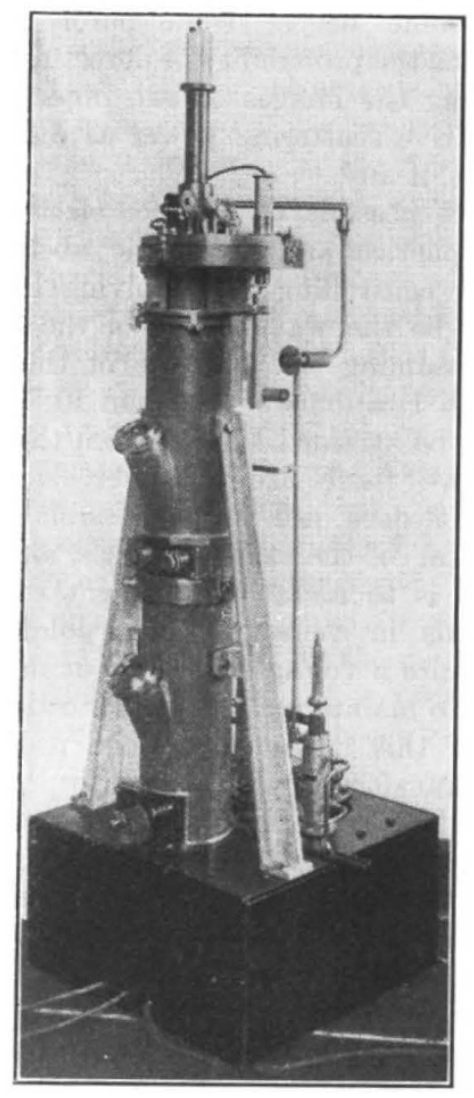

Fig. 2.

Electron microscope at the Imperial College, LoNDON.

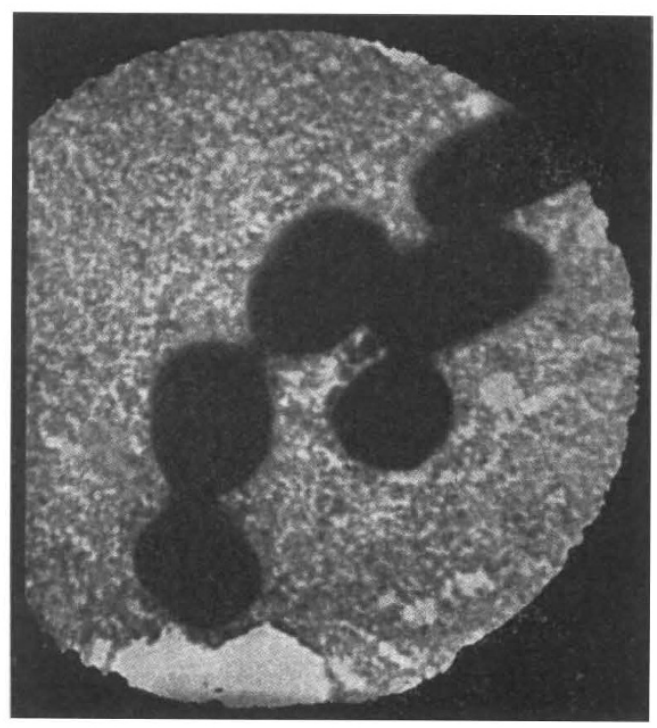

Fig. 3.

Micrococcus flavus; ELECTRON-OPTICAL MAGNIFICATION, 16,000. StruCtures NEAR THE COCCI DO NOT OCCUR IN THE SUPPORTING FILM; PHOTOGRAPHS OF PLAIN FILMS SHOW A COMPLETELY CLEAR GROUND. Photograph by von Borries, E. Ruska and $\mathrm{H}$. RUSKa, REPRODUCED BY COURTESY OF HERR $\mathrm{E}$. Ruska, of Stemens und Halske A.G.

borate, have been published by Beischer and Krause, after which the resolution of Amphipleura pellucida in dots seems a pedestrian performance. In order to photograph bacteria, they are dried by a special process on an extremely thin film of collodion, and can be photographed without any other special treatment. Photographs have been published (notably by von Borries, E. Ruska, and H. Ruska) showing various organisms such as Micrococcus flavus under extraordinarily high magnification, such as 16,000 ; one picture exhibits a fine structure, whatever its nature or origin, on the film around the dried bacteria, which could not possibly be observed in optical pictures (Fig. 3).

Delicate objects placed in an intense electron beam may of course suffer damage by heating and further by becoming so highly charged that electrostatic repulsive forces disintegrate the structure; it will therefore be understood that there is a great deal of special technique to be developed. The necessity of mounting the objects in a high vacuum is naturally a severe complication.

\section{THERMIONIC EMISSION}

The term 'electron microscope' has also been given to electron-optical systems designed to give images of surfaces which are self-emitters of electrons either by thermionic or by photo-electric action. Thus, for example, suitably activated 
surfaces of iron and nickel vary greatly in emissivity according to their crystalline state, and the changes in structure with change of temperature can be followed in an interesting way by projecting the images of such surfaces with suitable magnetic or electrostatic lenses, although the magnifications employed are not usually greater than one hundred, and there is little question of high resolution. The present article will not attempt to deal with these systems.

\section{Problems in Electron Microscopy}

Having briefly noticed the present state of progress in the subject, it may be of interest to indicate some of the problems which have to be faced in any attempt at further progress.

In an ordinary photomicrograph, a 'picture' of an opaque object is formed by absorption of the light, but when the object is only distinguished by a variation of refractive index with respect to the surrounding medium, the contrast in the image is secured, in many cases, because some of the light from particular regions of the object is deflected outside the aperture of the objective, and thus fails to reach the corresponding part of the image.

In the case of the electron radiation passing through a thin layer of matter, a large proportion of the moving electrons may not pass sufficiently close to any of the electrons or nuclei in the atoms of the film to suffer perceptible changes in energy or direction, since, as we now understand, the particles conceived as forming an atom occupy effectively but a small part of its dimensions as usually estimated. Other electrons may pass comparatively close to a relatively massive nucleus and swing around it in a hyperbolic orbit, suffering a comparatively large change of direction but, in comparatively many cases, inappreciable change of energy ; such collisions would be said to be of the 'elastic' type.

As has been shown by Bohr, however, the effect of the electrons present in the atom cannot be neglected; they are not to be regarded, in this connexion, as fixed, and thus can take up more or less kinetic energy from the particle in quantum units. In some cases the moving electron interacts so violently that one or more electrons are completely removed from the atom; the loss of kinetic energy may then be comparatively great and accompanied by a large change in direction of the incident particle. The collision is of the 'inelastic' type. In some cases (fluorescence) the energy-levels of the electrons of the material may be altered and light is emitted as they return to the normal configurations. In very thin films of matter the electrons are not likely to encounter more than a single nucleus sufficiently closely for appreciable scattering, and the most probable angle of deflection is proportional to the square root of the thickness, but with increasing thickness or with the presence of heavier atoms at particular regions, the mean scattering angle may be increased, sometimes by successive encounters. Some electrons may be completely trapped by the film, and if it is a non-conductor they remain as a charge on the surface.

In preparing objects such as bacteria for the electron microscope, they are usually mounted on very thin films of nitro-cellulose, and it is fortunate that the amount of scattering and spread of velocity caused by the film does not seem to be so great as to introduce serious trouble with spherical and 'chromatic' aberration. Potentials of about $75 \mathrm{kv}$. have usually been employed for this work. If an object is mounted on a film, it will scatter the radiation to a greater extent than the film support, and, as widely scattered electrons will fail to enter the narrow aperture of the objective, the object will show up as a dark region in the image. We have to remember, however, that the velocityspread caused by the mount may interfere to some degree with the definition, and the image will then differ to some degree from purely silhouette effects. Thus the probability of detecting an object or separating the images of two objects depends on the relative scattering power as distinct from the support, if any.

If it were possible to produce electron images free from spherical and 'chromatic' aberration, the only factor controlling the resolving limit would apparently be the wave-length of the de Broglie waves. Assuming the aperture of the corrected lens to be $0 \cdot 1$, a limit of less than $10^{-2} \mathrm{~cm}$. might apparently be attained. Apart from the questions raised above there are, however, serious difficulties. It does not seem possible to secure 'achromatism' of the electron lenses, and the only alternative is to make the incident radiation as homogeneous in velocity as possible. Various factors require a voltage of 50,000 or more, and it is difficult to maintain a perfectly steady electrical pressure of this magnitude. The usual circuits employ a transformer with valves to produce halfwave or full-wave rectification, together with as large a reservoir condenser as possible. The residual ripple can be reduced to one tenth of one per cent without undue trouble, and may be further reduced by filters.

When cold-cathode discharges are used, the current can be stabilized by a saturated diode valve, and the inhomogeneity is reduced to that caused by collision effects, etc., in the discharge tube supplying the electron stream. In all cases the perfection of the vacuum technique is essential, 
and conditions of the utmost constancy must be obtained. If necessary, complete stabilization of the supply for hot-cathode work can be attained by the use of a battery, but the high initial cost, maintenance, and rapid depreciation of such a battery present difficulties which will be well understood.

Assuming that the voltage spread can be sufficiently reduced, the question of the 'spherical' aberration of the 'lenses' comes into prominence. It is apparently not possible to design magnetic or electrostatic lenses which are free from this defect, and no known arrangement seems to do more than approach a certain minimum value. This question has been studied theoretically by Scherzer and also, on the basis of numerical calculations, by Rebsch, who reaches the conclusion that the limit of resolving power of electron-optical systems will always be set in this way at about ten to one hundred times the electron wave-length, instead of going below the wave-length itself as might be inferred from Abbe's optical formula. Whether or not this conclusion is just, the implied limitation does not exclude the possibility of opening up still further new fields in high-power microscopy.

Just as in ordinary optics, however, where more than half the serious troubles are due to faulty construction and lack of symmetry of the parts, the electron microscope is by no means easy to perfect in such respects. In the first place, the present instruments are given a length of about two metres in order to reach the required magnification in two stages, and moreover a complicated object-holder chamber and adjustable coils have to be included in the apparatus. It is thus scarcely practicable to secure a geometrical symmetry by merely screwing together a set of lathe-turned parts as is done in the telescope. Even if this were done, the electron beam would still be appreciably curved, unless the axis of symmetry coincided with the direction of the earth's magnetic field. Again, it is by no means easy to make an ironclad mag netic coil truly symmetrical, in the magnetic sense, about its mechanical axis. The residual irregularities in the winding, and probably especially the variation of magnetic resistance in the gaps of the soft iron sheathing, produce a lack of symmetry in the field; even more troublesome is the fact that the asymmetry varies with the varying current, especially when the magnetism of the sheath is approaching saturation. The perfection of such coils is an interesting problem.

For a number of reasons, therefore, the early instruments were built up with components arranged as symmetrically as possible, and the beam was sometimes persuaded along its required path by judicious use of bar magnets! In the instrument described by Martin, Whelpton, and Parnum, the necessary control was obtained in a less objectionable way by the use of paired coils in the Helmholtz manner. This instrument, in use at the Imperial College, is now so arranged that the objective coil can be adjusted both in tilt and lateral position, and further, the condenser and projection coils can be adjusted laterally, all from outside the vacuum.

Neglecting the residual asymmetry of a coil which is not, after all, very large, the system of initial adjustment depends on the fact that if any 'object' is not on the axis of a coil, the image will move in a radial direction around the axis when the current in the coil is varied. The finer centring adjustments may require the deliberate use of inhomogeneity in the beam velocities. Failing perfect centration, very serious aberrations in the image will correspond to lateral chromatic spreading of the image points and 'coma', even in the centre of the picture.

A major part of the experimental work at the Imperial College has been devoted to a study of the 'optical' adjustments of the system in the above sense, and it is hoped that when more perfect electrical equipment is available, the experience thus gained will make it possible to obtain the optimum results.

A word may be added as to the relations of the electron microscope to the research methods of 'X-ray' and 'electron diffraction' analysis. It is true that where some degree of regularity exists in the structure of matter, these powerful and beautiful methods can be used with confidence to elucidate the atomic pattern, just as diffraction spectra appearing in the optical microscope may prove the existence of a structure which still cannot be resolved by the instrument. It is the possibility that the electron microscope may prove a means of examining irregularities and discontinuities in structures which makes it seem so promising, even if the method cannot deal with elements so small as those of crystal lattices.

On the analogy of the Abbe principle, it may be said that if the aperture and corrections of the 'lenses' could be so far improved as to include the electron diffraction maxima from a crystalline structure without incurring appreciable aberration, the very elements of the lattice would be resolved in the picture. That achievement may at present be quite outside the limits of apparent probability, but the subject is still in its infancy and at a stage corresponding to the period in the history of the optical microscope before the achromatic lens was invented. We may learn from the experience of those days not to be over-confident in setting limitations to what is possible. 